\title{
Topologically protected spin diffusion and spin generator using chalcogenide superlattices
}

\author{
Junji Tominaga $\mathbb{D}^{1 凶}$, Noriyuki Miyata ${ }^{1}$, Satoshi Sumi ${ }^{2}$, Hiroyuki Awano ${ }^{2}$ and Shuichi Murakami ${ }^{3}$
}

Spintronics is expected to be the basis for future ultra-low-energy nanoelectronic devices. To operate such devices at room temperature, amplifiers, batteries, capacitors, as well as spin current sources are required. Here we report a chalcogenide superlattice composed of $\mathrm{GeTe}$ and $\mathrm{Sb}_{2} \mathrm{Te}_{3}$ layers that have a topologically protected spin diffusion length exceeding $100 \mu \mathrm{m}$ at room temperature. A spin generator is demonstrated by combining magnetic injectors (TbFeCo) with this superlattice. The spin current was found to increase exponentially with the number of superlattice periods. We used this effect to demonstrate a 15-fold increase in the spin current. In addition, spin rectification is possible by growing the superlattice layers with atomic-level thickness accuracy. The reported chalcogenide superlattice spin generators and rectifiers open new opportunities to design low-energy spintronic integrated circuits and quantum computers.

npj 2D Materials and Applications (2020)4:22; https://doi.org/10.1038/s41699-020-0157-8

\section{INTRODUCTION}

Spintronics is key to realizing ultra-low power electronics ${ }^{1-9}$. An example of spintronic device is a magnetoresistive nonvolatile memory. Spintronic devices usually consist of three parts: a spin injector, a spin detector, and a medium to transport spins. The injector and detector are usually made of a ferromagnetic material and a metal with large spin-orbit coupling, whereas the transport medium is generally a metal or a semiconductor. Although injected spin current from a ferromagnetic material diffuses through the transport medium, and is rapidly attenuated due to scattering. The spin diffusion length at room temperature is generally less than $1 \mu \mathrm{m}$ in metals or semiconductors ${ }^{10}$. A longer spin diffusion length is required for integrating spintronic devices using a single spin source or battery. Therefore, a technology that extends the spin diffusion length is expected to revolutionize lowenergy electronic devices.

Spintronics devices can be enhanced by exploiting the discovery of topological insulators (TIs) and other related materials, such as Dirac and Weyl semimetals (DS and WS $)^{11-16}$. Importantly, the spin in Tls is protected from scattering, even at room temperature, by spatial inversion and time-reversal symmetries. However, spin transport in Tls is restricted to edges in twodimensional structures and to surfaces in three-dimensional (3D) structures. This low dimensionality limits the current that can be transported.

Recently, chalcogenide topological superlattices (SLs) have attracted attention, because they can be used to transport spin using $p$-electrons ${ }^{17-22}$. These SLs consist of stacked chalcogenide normal insulator (NI) and TI thin layers. Similar SLs, which consist of $\mathrm{GeTe}(\mathrm{NI})$ and $\mathrm{Sb}_{2} \mathrm{Te}_{3}(\mathrm{TI})$, have also been applied to nonvolatile phase-change memory ${ }^{17,23}$. The ferroelectric phase, or simply "Ferro-phase", of these SLs is sensitive to magnetic fields at room temperature. This is interesting, because it does not contain any magnetic elements ${ }^{18,19,22,24}$. The origin of the magnetic sensitivity is due to the $p_{z}$-electrons of $\mathrm{Ge}$ and Te atoms in the $\mathrm{Ge}_{2} \mathrm{Te}_{2}$ layers of the $\mathrm{SL}^{19}$. Until now, these SLs have only been applied to nonvolatile phase-change memory devices. However, the fact that the Ferro-phase is a WS implies that they may also be important in spintronics devices and hybrid phase-change spintronics universal memories $^{25,26}$.

In this study, we show that the spin diffusion length of the $\mathrm{Ge}_{2} \mathrm{Te}_{2}-\mathrm{Sb}_{2} \mathrm{Te}_{3} \mathrm{SLs}$ is over $100 \mu \mathrm{m}$ at room temperature. Moreover, we demonstrate how this topologically protected spin diffusion length can be used in prototype spin generators and rectifiers that operate over hundreds of micrometers. Indeed, the long spin diffusion length allowed these prototypes to be patterned using inexpensive and simple techniques without photo- or election beam lithography.

\section{RESULTS AND DISCUSSION}

Spin transport length of $\mathrm{Ge}_{2} \mathrm{Te}_{2}-\mathrm{Sb}_{2} \mathrm{Te}_{3} \mathrm{SL}$

The SLs used in this work consist of layers of $\mathrm{Sb}_{2} \mathrm{Te}_{3}$ and $\mathrm{Ge}_{2} \mathrm{Te}_{2}$ atomic units ${ }^{18,19,22,25-28}$. These layers are alternatively grown as thin films (see "Methods"). The $\mathrm{Sb}_{2} \mathrm{Te}_{3}$ layer is covalently bonded with a Te-Sb-Te-Sb-Te atomic stacking sequence, whereas the units are weakly held together by the van der Waals (vdW) force. On the other hand, the $\mathrm{Ge}_{2} \mathrm{Te}_{2}$ layer has three possible polymorphs with atomic sequences: Ge-Te-Te-Ge, Te-Ge-Ge-Te, and Te-Ge-Te-Ge (Ge-Te-Ge-Te) ${ }^{28}$. These phases are respectively called Petrov, inverted-Petrov, and Ferro. The $\mathrm{Ge}_{2} \mathrm{Te}_{2}-\mathrm{Sb}_{2} \mathrm{Te}_{3}$ layers are bonded by the $\mathrm{vdW}$ interaction when both units are terminated with Te atoms. This happens when the $\mathrm{Ge}_{2} \mathrm{Te}_{2}$ is in the inverted-Petrov and Ferro-phase. However, in the case of the Petrov phase, the interface has hetero-terminations (Ge and Te), which are covalently bonded.

The Ferro-phase is thermodynamically most stable at more than $500 \mathrm{~K}$ and therefore most likely to exist, when the SL is grown at around $520 \mathrm{~K}^{19}$. However, as the Ferro-phase is cooled to room temperature, the phase transition converts the Ferro-phase into the Petrov and inverted-Pertov phases, and the SLs lose magnetic sensitivity ${ }^{28}$. This is due to the appearance of spatial inversion

\footnotetext{
${ }^{1}$ Systematic Materials Design Group, Device Technology Research Institute, National Institute of Advanced Industrial Science and Technology (AIST), Tsukuba, Japan. ${ }^{2}$ Memory Engineering Laboratory, Department of Advanced Science and Technology, Toyota Technological Institute, Nagoya, Japan. ${ }^{3}$ Department of Physics, School of Science, Tokyo Institute of Technology, Tokyo, Japan. ${ }^{凶}$ email: j-tominaga@aist.go.jp
} 
symmetry in the Petrov and inverted-Petrov phases, which is not present in the Ferro-phase. The electronic band structure of the $\mathrm{Ge}_{2} \mathrm{Te}_{2}-\mathrm{Sb}_{2} \mathrm{Te}_{3} \mathrm{SL}$ in the Ferro-phase has been reported by several groups. Due to the break of the spatial inversion symmetry, the degenerate bands are lifted, except those at the Kramers points in the reciprocal space ${ }^{18,29,30}$. Moreover, it was reported that band splitting induces a large Rashba effect ${ }^{19}$. Therefore, the Ferrophase is magnetically sensitive, whereas the Petrov and invertedPetrov phases are not. It is noteworthy that the Ferro-phase is also a WS 25,26 .

To increase the amount of the Ferro-phase in the SL the "thermal-annealing under magnetic field (TAUM)" process was proposed $^{22}$. However, TAUM processing requires temperature above the $470 \mathrm{~K}$, which is the above the Curie temperature of many ferromagnetic materials, thus rendering it unsuitable for spin injectors. To solve this problem we have used a new method to grow Ferro-phase SLs (see "Methods"). A high-resolution and atomically resolved transmission electron microscope image of a typical SL in the Ferro-phase is given in Ref. ${ }^{28}$. We used this new SL throughout the present work.

Herein we first demonstrate the spin transport properties of the $\mathrm{SL}$ at room temperature. Device-A was fabricated with TbFeCo $(80 \mu \mathrm{m}$ width) and $\mathrm{Pt}(80 \mu \mathrm{m}$ width) electrodes, which were directly deposited on the SL through shadow masks. A microscope

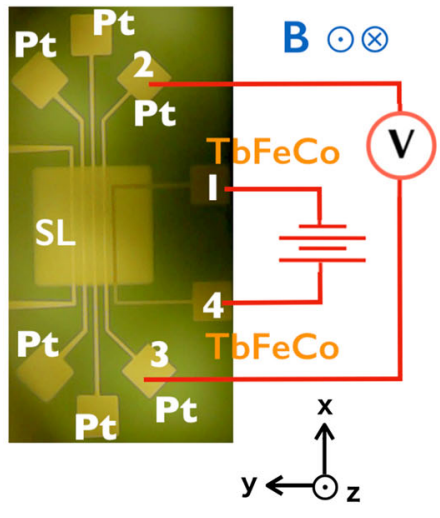

(a)

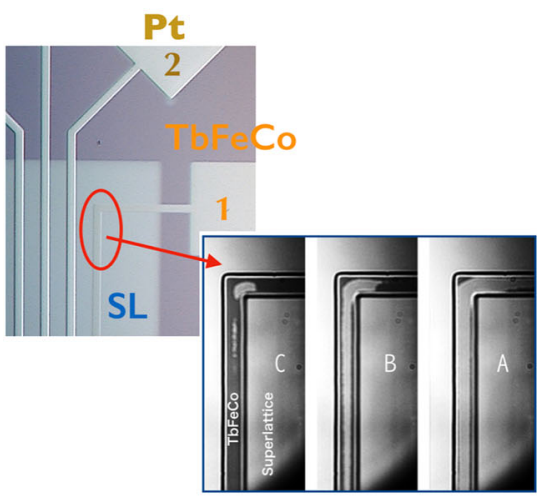

(c) photograph of the device and the measurement setup are shown in Fig. 1a. TbFeCo is ferromagnetic and it was used as the spin injector. It has perpendicular magnetic anisotropy to the film surface $^{31}$. This particular ferromagnetic alloy was widely used in magneto-optical recording media (disk) in the 1990s. TbFeCo films can be deposited into an amorphous structure by sputtering at room temperature. The lack of grain boundaries in the amorphous structure greatly increases the Kerr rotation signal to noise ratio ${ }^{32}$. For this reason, it can be expected in this work as well that the noise from the grain interface can be reduced, rather than using a crystalline ferromagnetic film. On the other hand, the Pt electrodes are used to convert spin current $\left(j_{s}\right)$ into a charge current $\left(j_{c}\right)$ by the inverse spin Hall (ISH) effect ${ }^{33-35}$. The anisotropic magneto-resistance (AMR) of the TbFeCo film with a $50 \mathrm{~nm}$ thickness is shown in Supplementary Fig. 1. A SL structure composed of six $\left(\mathrm{Ge}_{2} \mathrm{Te}_{2}\right) /\left(\mathrm{Sb}_{2} \mathrm{Te}_{3}\right)$ bilayer periods sandwiched between the upper and lower $5 \mathrm{~nm}$-thick $\mathrm{Sb}_{2} \mathrm{Te}_{3}$ layers was used as the spin-transfer layer.

The ISH voltages $\left(V_{\mathrm{ISH}}\right)$ obtained at a $100 \mu \mathrm{m}$ distance are shown in Fig. $1 \mathrm{~b}$. In the vicinity of the magnetic domain transition in the spin injector (TbFeCo), $V_{\text {ISH }}$ signals are clearly changed (Fig. 1c) and reversed in the opposite magnetic field sweeping. Moreover, the polarity of the $V_{\mathrm{ISH}}$ signal is reversed when the direction of the $j_{\mathrm{C}}$ is reversed. The $V_{\mathrm{ISH}}$ linearly increases with the $j_{\mathrm{C}}$ (Supplementary

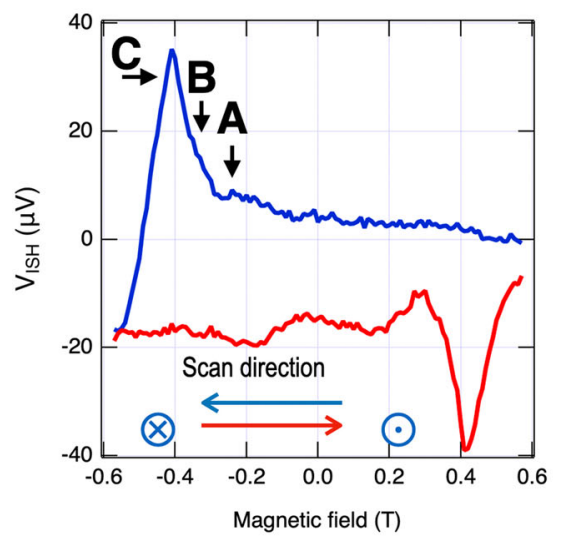

(b)

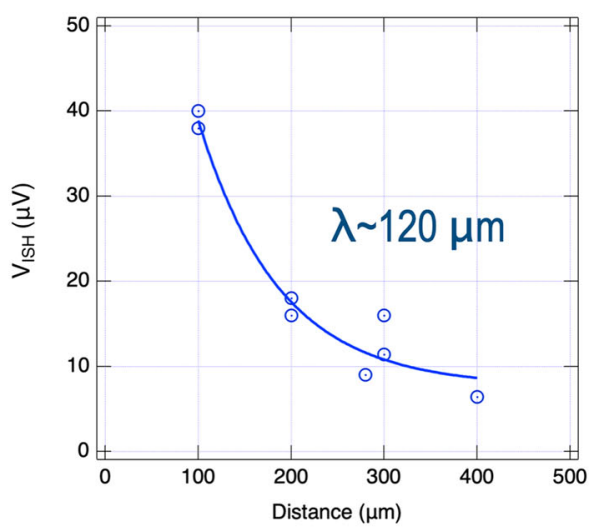

(d)

Fig. 1 Device structure and inverse spin Hall performance. a Optical microscopic views (zoom-in and out) of Device -A. The charge current $j_{c}$ flows between the TbFeCo electrode pads, 1 and 4, and the ISH voltage $\left(V_{I S H}\right)$ was detected between the adjacent Pt electrode pads, 2 and 3. All electrode widths were fixed at $80 \mu \mathrm{m}$. The magnetic field was applied normal to the sample surface. $\mathbf{b} V_{\text {ISH }}$ as a function of magnetic field at a $100 \mu \mathrm{m}$ electrode distance, using a spin current, $j_{c}$, of $2 \mathrm{~mA}$. Prior to sweeping the magnetic field, the TbFeCo electrode was magnetized at $+0.55 \mathrm{~T}$, where $V_{\mathrm{ISH}}$ was set to zero. c Magnetic domain transition optically observed at the corner of the TbFeCo injector near A, B, and C points in $\mathbf{b}$. $\mathbf{d} j_{\mathrm{s}}$ diffusion length (1/e), $\lambda$, which was estimated using several devices with different electrode distances. 


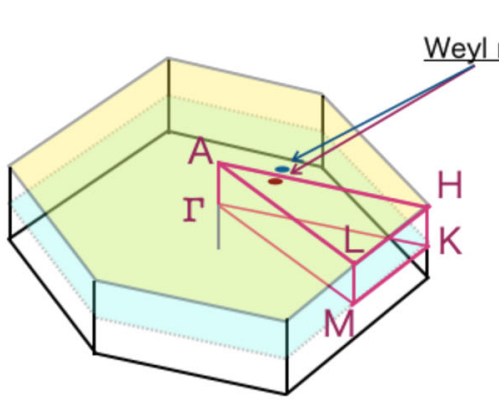

(a)

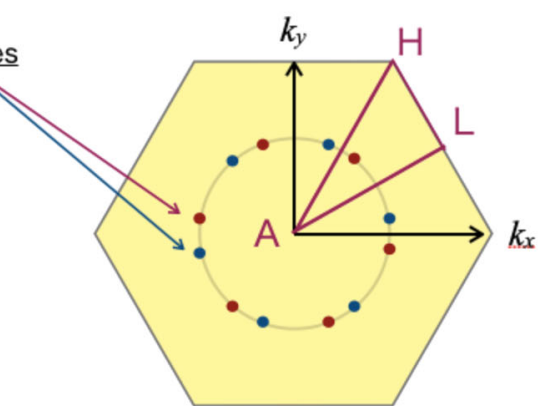

(b)

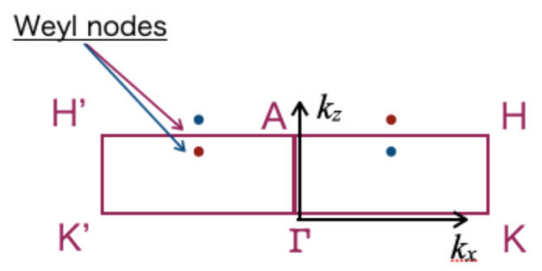

(c)

Fig. 2 Configuration of the Weyl nodes in $\mathbf{G e}_{2} \mathbf{T e}_{2}-\mathrm{Sb}_{2} \mathrm{Te}_{3} \mathrm{SL}$. a The hexagonal reciprocal lattice of the SL. Pairs of effective magnetic monopoles (red and blue circles) are located along the A-H line on the $k_{z}=\pi / c$ plane. $\mathbf{b}$ The top view. The 12 Weyl nodes exist and every 2 nodes are magnetically coupled. $\mathbf{c}$ The side view. The coupled monopoles have an offset to the $k_{z}=\pi / c$ plane ${ }^{25}$.

Fig. 2). The $V_{\mathrm{ISH}}$, however, exhibits an approximately exponential dependence on the electrode separation. A similar is found for semiconductor and metal transport media ${ }^{10,35}$. The $j_{\mathrm{s}}$ diffusion length, $\lambda$, was estimated to be $120 \mu \mathrm{m}$ (see Fig. 1d). These experimental results show that the SL can transport $j_{s}$ over a distance of $200 \mu \mathrm{m}$ at room temperature. It is noteworthy that $V_{\mathrm{ISH}}$ is very sensitive to the SL layer thicknesses of $0.8 \mathrm{~nm}$ for $\mathrm{Ge}_{2} \mathrm{Te}_{2}$ and $1.0 \mathrm{~nm}$ for $\mathrm{Sb}_{2} \mathrm{Te}_{3}$. This is discussed later, whereas the magnetic sensitivity of the SL was also reported in our previous work $^{22}$.

\section{Spin amplification}

Theoretically, it is known that $j_{\mathrm{s}}$ in non-magnetic media, such as semiconductors or metals, relies on the difference between the electrochemical potentials of up-spin $\left(\mu_{\uparrow}\right)$ and down-spin $\left(\mu_{\downarrow}\right)$ states at the Fermi level, and $j_{s}$ is given by

$j_{\mathrm{s}}=\frac{\hbar \sigma}{2 e^{2}} \nabla\left(\mu_{\uparrow}-\mu_{\downarrow}\right)$

where, $\hbar, e$, and $\sigma$ are Planck constant, electron charge, and conductivity, respectively. However, $j_{s}$ is exponentially reduced by scattering after injection. Therefore, in developing the spin current amplifier, it is important to increase the chemical potential difference (spin accumulation, $\mu_{\mathrm{s}}=\mu_{\uparrow}-\mu_{\downarrow}$ ) at both edges in the injection electrode ${ }^{35}$. On the other hand, in Tls the mechanism of the $j_{s}$ transport is different from semiconductors and metals due to time-reversal symmetry. As reported by Kim et al. ${ }^{25}$, the SL with the Ferro-phase is a WS with 12 Weyl nodes. These nodes are arranged into six pairs and distributed around the $\mathrm{A}-\mathrm{H}$ highsymmetry lines in reciprocal space. This is depicted in Fig. $2^{25}$.

The Weyl-pair nodes are chiral and respectively correspond to effective magnetic monopoles ( $\mathrm{N}$ and $\mathrm{S}$ ), which are located around the A-point along the six $\mathbf{A}-\mathbf{H}$ lines. As all the effective magnetic monopoles can satisfy time-reversal symmetry with other monopoles located at the opposite sites beyond the A-point, the spin direction is not constrained on the $k_{x}-k_{y}$ plane. This is different from the Rashba bands that occurred around the $\Gamma$-point. Notice that the Weyl nodes are not located at $k_{z}=0$, but at $\pi / c$. Thus, near the Weyl bands an electron has a momentum in $k_{\mathrm{z}}$. Furthermore, the Weyl-pair bands share spin-up and spin-down electrons. Thus, the WS-SL has two independent spin transport channels (up and down), and electron back scattering is prohibited by the time-reversal symmetry and the chirality of the Weyl nodes.

In Tls, instead, Berry phases and curvatures play a role in the transport. The Berry phases for $j_{c}$ and $j_{s}$ are, respectively, described in a simple two-band system with the $\mathbf{S}_{\mathbf{z}}$ conservation as,

$$
\mathbf{A}^{\mathbf{c}}(\mathbf{k})=-i\left\{\left\langle u_{\text {up }}(k)\left|\nabla_{k}\right| u_{\text {up }}(k)>+<u_{\text {down }}(k)\left|\nabla_{k}\right| u_{\text {down }}(k)>\right\}\right.
$$

and

$$
\mathbf{A}^{\mathbf{S z}}(\mathbf{k})=-i\left\{\left\langle u_{\text {up }}(k)\left|\nabla_{k}\right| u_{\text {up }}(k)>-<u_{\text {down }}(k)\left|\nabla_{k}\right| u_{\text {down }}(k)>\right\}\right.
$$

where $\mid u_{\text {up }}(k)>$ and $\mid u_{\text {down }}(k)>$ are the wavefunctions in each spin state. Integration of the Berry curvature $\nabla \times \mathbf{A}^{\mathbf{c}}(\mathbf{k})$ over the Brillouin zone is zero, whilst that of $\nabla \times A^{\mathbf{S z}}(\mathbf{k})$ is not. This implies that in terms of time-reversal symmetry $j_{s}$ can be tightly protected in Tls. Moreover, as the $k_{z}$ is non-zero at the Weyl nodes in the SL, the spin transport may spontaneously induce spin polarization.

When spins are polarized in the z-direction (normal to the SL layers), electrons are injected to the SL by flowing $j_{c}$ in the TbFeCo electrode (in the $x$-direction) and $j_{s}$ flows in the $y$-direction. If the SL is a uniform WS, as discussed above, the $j_{\mathrm{s}}$ polarity is switched with the $j_{c}$ polarity. Therefore, when the $j_{c}$ flows in the opposite direction in each of the multiple ferromagnetic electrodes, the $j_{s}$ alternately switches polarity in the $+y$ and $-y$ directions. If the electrode separations are shorter than the characteristic spin diffusion length, spin accumulation $\mu_{\mathrm{s}}$ can be increased by a factor of two or more. As a result, the increased chemical potential between the electrodes leads to an increase in the $j_{s}$ flowing out of the electrodes. In other words, such a device could function as a spin amplifier.

To confirm this scenario, we prepared three different samples (Device- $B,-C$, and -D) and compared the $V_{\text {ISH }}$ intensities, as shown in Fig. 3a. In Device- $B$, three spin injectors were connected in parallel, whereas in Device- $C$ the injectors were connected in series. $V_{I S H}$ intensities of Device- $B,-C$, and $-D$ were compared against the $j_{c}$ flowing per injector. As clearly seen in Fig. $3 \mathrm{~b}$, the $V^{C}{ }_{\text {ISH }}$ of Device-C is amplified by a factor of two relative to the control device (D), $V^{D_{\text {ISH }}}$ (gray). The results are in good agreement with our theoretical prediction. When the electrode distances are changed from 200 to $100 \mu \mathrm{m}, V_{\mathrm{ISH}}$ is further increased by a factor 

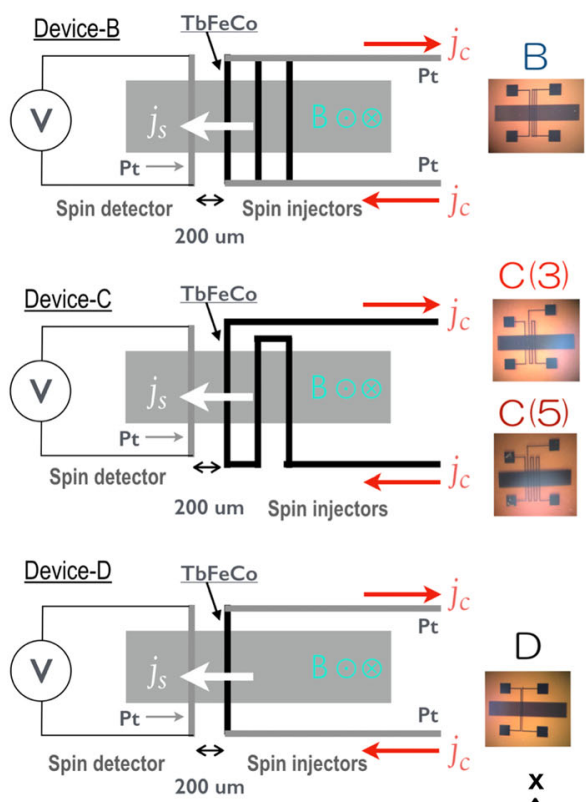

(a)

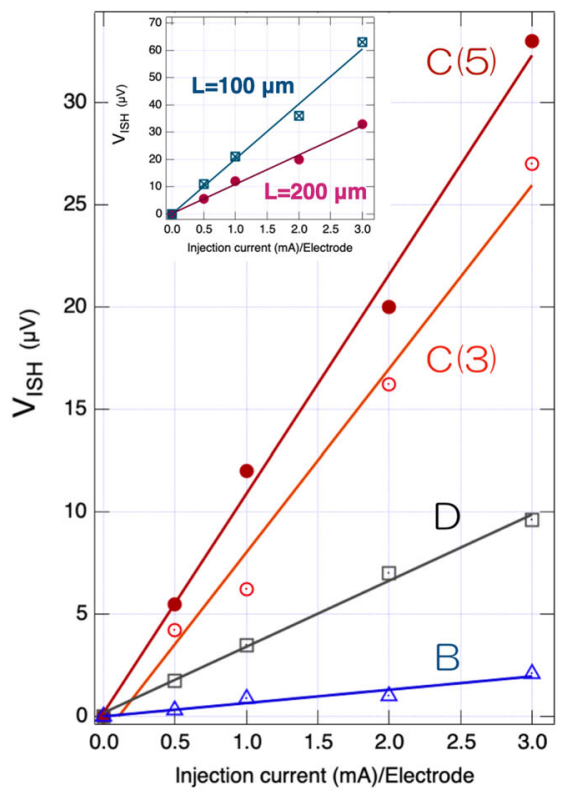

(b)

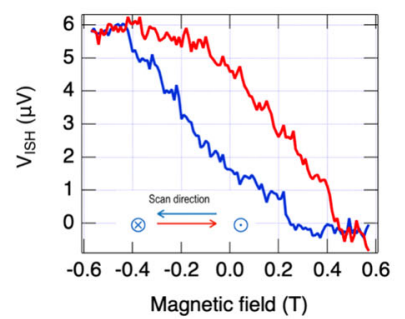

(c)
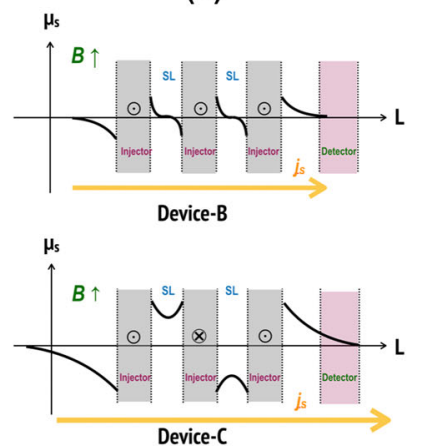

(d)

Fig. 3 Device structures designed for spin amplification and the performance. a Three different devices were fabricated. Device-B had three spin injectors connected in parallel, Device-C had three or five spin-injectors connected in series, and Device-D was a control with a single injector. The electrode separation distance were fixed at $200 \mu \mathrm{m}$ and the electrode width was $80 \mu \mathrm{m}$. $\mathbf{b} j_{\mathrm{c}}$ dependence of $V_{\mathrm{ISH}}$ signals of DeviceB (blue), -C (red), and -D (gray), where $j_{c}$ was normalized per injector. The inset compares the signal voltage of Device-C (five electrodes) for electrode distances of 100 and $200 \mu \mathrm{m}$. c The $V_{\text {ISH }}$ hysteresis loop for Device-C (three electrodes) for $j_{C}=1.0 \mathrm{~mA}$. d A schematic showing spin accumulation $\boldsymbol{\mu}_{\mathrm{s}}$ in Device-B and -C. In Device-B, as $j_{\mathrm{c}}$ flows in parallel through each interface, $\boldsymbol{\mu}_{\mathrm{s}}$ between the electrodes is weakened due to the opposite spin polarities. As a result, the outer $\boldsymbol{\mu}_{\mathbf{s}}$ is relatively weak. In Device-C, the $j_{\mathrm{c}}$ direction of the central electrode is opposite to the outer electrode; thus, the same spins with $\uparrow$ or $\downarrow$ polarity are accumulated and this enhances $\boldsymbol{\mu}_{\mathbf{s}}$ between the electrodes. As a result, the $j_{\mathrm{s}}$ flows with a high intensity. $\mathrm{B} \uparrow, \mathrm{SL}, \odot$, and $\otimes$ indicate the magnetic field direction, $\mathrm{SL}$, and $j_{\mathrm{c}}$ directions, respectively. Dotted lines indicate boundaries between injector edges and SLs. Black curves and yellow arrows are $\boldsymbol{\mu}_{\mathrm{s}}$ polarity and $j_{\mathrm{s}}$ flow, respectively.

of two (Fig. 3b inset). The $V_{\text {ISH }}$ hysteresis curve is shown in Fig. 3c. Moreover, when increasing the number of the injectors from three to five in Device-C, $V_{\text {ISH }}^{C}$ increases by a factor of three.

In contrast, the $V^{B}{ }_{\text {ISH }}$ of Device- $B$ is decreased by a factor of five. In Device- $B, j_{s}$ flows in the same direction in the three parallel electrodes as indicated by the white arrow in Fig. 3a. Assuming that the density of the up-spin is larger than that of the downspin, the up-spins flow in the $+y$ direction from the left edges in the electrodes, whereas the down-spins flow in the $-y$ direction from the right edges. Therefore, there is a net $j_{\mathrm{s}}$ flow in the $+y$ direction. However, in the spaces between the electrodes, the presence of opposite spins reduces the spin potential. As a result, the overall $j_{s}$ flowing out of the left edge in the left electrode is not expected to be greater than that of the single electrode case (Device-D) $)^{35}$. The evidence of the small $V_{\text {ISH }}^{B}$ implies that opposite spins survive scattering, reducing the overall $\mu_{\mathrm{s}}$ between the injectors. The difference of the spin accumulation $\mu_{\mathrm{s}}$ in the injector configurations is depicted in Fig. $3 d$.

These experimental results imply that the spins with different polarities are alternatively accumulated between the injectors in the Device-C. If probes were contacted between the injectors, one would extract a much larger $j_{\mathrm{s}}$ corresponding to the $\mu_{\mathrm{s}}$. The device behavior is reminiscent of a spin capacitor, or spin storage device connected in series ${ }^{36,37}$.

$V_{\text {ISH }}$ can further be amplified by increasing the number of the $\mathrm{Ge}_{2} \mathrm{Te}_{2}$ layers in the $\mathrm{SL}$, as the Ferro-phase is a WS. The linear bands (Weyl nodes) bridging the bulk gap are mainly composed of $p$ electrons of Sb and Te near the vdW gap space (Supplementary Fig. 3). In contrast, the linear bands (Dirac point) in the inverted-Petrov at the $\Gamma$-point, which is a DS, are composed of $p$-electrons of Ge and Te within the $\mathrm{Ge}_{2} \mathrm{Te}_{2}$ layers (Supplementary Fig. 4) ${ }^{19,25,26}$. In the SL, $\mathrm{Ge}_{2} \mathrm{Te}_{2}$ and $\mathrm{Sb}_{2} \mathrm{Te}_{3}$ units, and the vdW gap spaces are alternatively stacked in the z-direction with a period of $1.8 \mathrm{~nm}$, which is much shorter than the spin diffusion length shown in Fig. 1d. Therefore, the attenuation of the injected spins from the ferromagnetic electrodes is negligible. In addition, since in the SL, the $k_{z}$ of the Weyl nodes has an offset, the spin diffusion will increase with the number of the period. These multiple electrons confined within interfaces or the $\mathrm{Ge}_{2} \mathrm{Te}_{2}$ layers play a role as spin transfer channels. Figure $4 a$ shows this effect using Device-C with three spin injectors. The amplification exponentially increases with the number of the $\mathrm{Ge}_{2} \mathrm{Te}_{2}$ layers. Indeed, a five-fold increase in $j_{s}$ relative to a device with a single injector for a SL with 12 bilayer periods. The SL $V_{\mathrm{ISH}}$ amplification gain is compared against a $4.8 \mathrm{~nm}$-thick single layer of GeTe and with single layer of $6.0 \mathrm{~nm}$ thick $\mathrm{Sb}_{2} \mathrm{Te}_{3}$ layer. These layer thicknesses correspond to an equivalent SL with $n=6$ periods. These single layers showed $<100 \%$ gain. In the devices, as the transport channels are limited to one (bulk for GeTe) or two (surfaces for $\mathrm{Sb}_{2} \mathrm{Te}_{3}$ ) respectively, both $V_{\mathrm{ISHs}}$ are not amplified with the thickness. These results clearly imply that the number of the alternatively stacked $\mathrm{Sb}_{2} \mathrm{Te}_{3}$ and $\mathrm{Ge}_{2} \mathrm{Te}_{2}$ layers, as shown in Fig. $4 \mathrm{~b}$ are responsible for the $V_{\mathrm{ISH}}$ amplification effect. In other words, the spin behavior in the SLs is attributed to the periodicity effect on the electronic band structure. We attribute the long spin diffusion length observed in our devices to the multiple spin transport channels that form due to the periodic arrangement of $\mathrm{Ge}_{2} \mathrm{Te}_{2}$ layers and the Ferrophase $\mathrm{SL}$, which was grown with a high crystal quality ${ }^{28}$. 


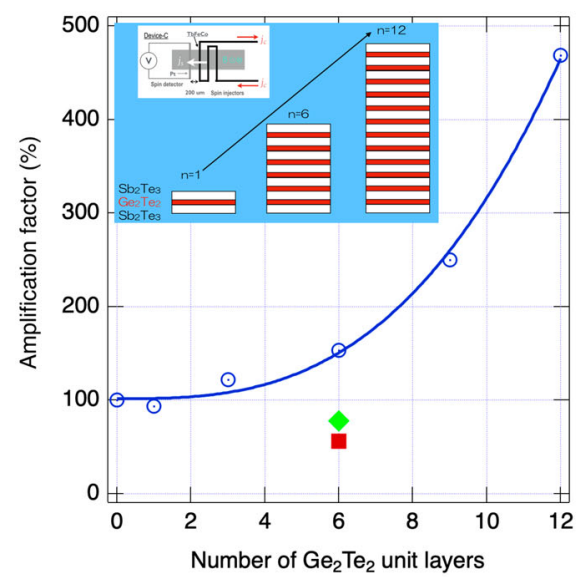

(a)

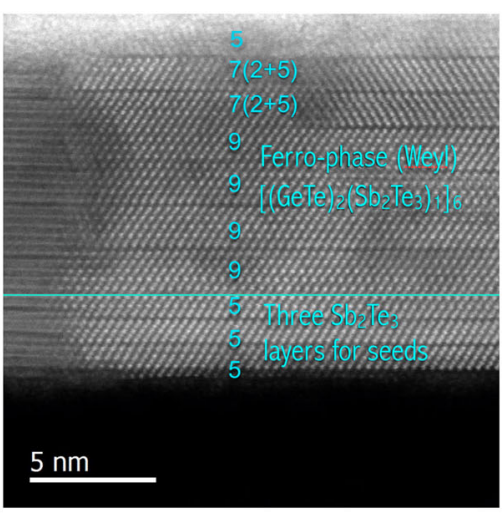

(b)

Fig. 4 Amplification by the SL periodicity. a Relationship between the number of $\mathrm{Ge}_{2} \mathrm{Te}_{2}$ unit layers and $V_{\mathrm{ISH}}$ again, using Device-C (blue), where $j_{c}$ and electrode distance were fixed to $2 \mathrm{~mA}$ and $200 \mu \mathrm{m}$, respectively. $V_{\mathrm{ISHs}}$ of a $6.0 \mathrm{~nm}$-thick $\mathrm{Sb}_{2} \mathrm{Te}_{3}$ layer (green diamond) and of a $4.8 \mathrm{~nm}$-thick GeTe layer (red square) corresponding to the total thickness of the SL with $\mathrm{n}=6$ are shown together. Inset: a schematic of the SL stacking structures. b A cross-sectional view of a six-periodic SL composed of the $\left(\mathrm{Ge}_{2} \mathrm{Te}_{2}\right) /\left(\mathrm{Sb}_{2} \mathrm{Te}_{3}\right)$ bilayers using a transmission electron microscope (TEM). At every five $\left(\mathrm{Sb}_{2} \mathrm{Te}_{3}\right)$, seven $\left(\mathrm{Sb}_{2} \mathrm{Te}_{3}+\mathrm{GeTe}\right)$, and nine $\left(\mathrm{Sb}_{2} \mathrm{Te}_{3}+\mathrm{GeTeGeTe}\right)$ atoms, a vdW gap are inserted to get the energy minimum.

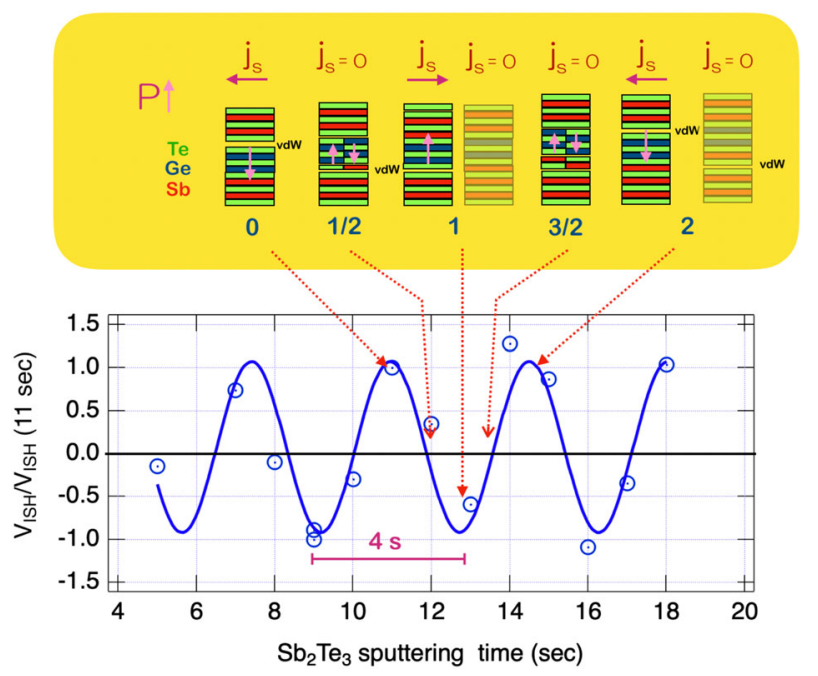

Fig. $5 \quad V_{\text {ISH }}$ polarity change with additional sputtering time of $\mathrm{Sb}_{2} \mathrm{Te}_{3}$ in Device-A. $j_{c}$ and the electrode distance were fixed to $2 \mathrm{~mA}$ and $200 \mu \mathrm{m}$, respectively. Hence, $j_{\mathrm{s}} \leftarrow$, and $\rightarrow$ are spin current and the directions. $P$ and $\uparrow \downarrow$ are the electric dipole direction of a $\mathrm{Ge}_{2} \mathrm{Te}_{2}$ layer. $V_{\mathrm{ISH}}$ are normalized using $V_{\mathrm{ISH}}$ at 11 seconds corresponding to the exact thickness of one $\mathrm{Sb}_{2} \mathrm{Te}_{3}$ layer, corresponding to the exact $\left(\mathrm{Ge}_{2} \mathrm{Te}_{2}\right) /\left(\mathrm{Sb}_{2} \mathrm{Te}_{3}\right)$ thickness, which is shown as "0" state in the top yellow panel. Additional half to two-layer models due to $\mathrm{Sb}$ and $\mathrm{Te}$ atoms are also shown as "1/2", "1", 3/2," and "2", respectively.

\section{Spin rectification}

Finally, we explain the factors that determine the $j_{s}$ polarity. Figure 5 shows the polarity of $V_{I S H}$ observed whilst sweeping the direction of the magnetic field (Fig. 1b). The $j_{s}$ polarity switches when layer thickness changes by that of a single layer of $\mathrm{Te}$ or $\mathrm{Sb}$ atoms. When the bottom $\mathrm{Sb}_{2} \mathrm{Te}_{3}$ layer is followed by the Ferro-phase of $\mathrm{Ge}_{2} \mathrm{Te}_{2}$, the Ge-Te-Ge-Te atomic order is stably formed, which is corresponds to the " 0 " state in the yellow panel of Fig. ${ }^{28}$. In the " 0 " state, as the SL breaks the spatial inversion symmetry, $j_{s}$ flows. In this example we assume, the direction is to the left. Since the Ge-Te-Ge-Te atomic order is ferroelectric, the electric dipole is directed from top to bottom and the vdW gap position moves up to the interface with the second $\mathrm{Sb}_{2} \mathrm{Te}_{3}$ layer. When a half layer is formed on the completed $\mathrm{Sb}_{2} \mathrm{Te}_{3}$ layer ("1/2" state in the panel), both $\mathrm{Sb}$ and Te atoms may terminate the structure. However, the mixture cancels the electric dipoles. When the first layer is formed ("1" state in the panel) next, only the Te layer can break the symmetry, whereas the Sb layer remains unchanged. It is noteworthy that the dipole direction is reversed, the " $3 / 2$ " state is the same as " $1 / 2$ " state, and the $j_{s}$ of the " 2 " state has the same direction as that of state " 0 ," as the vdW gap is moved up to the top of the $\mathrm{Ge}_{2} \mathrm{Te}_{2}$ layer. In the case that the vdW gap position is adjacent to the $\mathrm{Ge}_{2} \mathrm{Te}_{2}$ layer, $V_{I S H}$ becomes high. Density functional calculations demonstrated that the gap position and the dipole direction shift the Fermi level up or down near the Weyl points in the band structure.

From these analysis, it is interesting to consider whether the $v d W$ gap plane is terminated using either-Ge-Te or -Sb-Te. As Kim et al. ${ }^{25}$ suggested, the surface termination of the surface by -Ge-Te or -Sb-Te determines the Weyl node properties. As the energy level of the Weyl node pairs has an offset in the $\pm k_{z}$ direction, the interface termination presumably affects the spin polarization and the $j_{s}$ polarity. This effect remains to be clarified in a detailed analysis of Weyl semimetals.

In summary, $\mathrm{Ge}_{2} \mathrm{Te}_{2}-\mathrm{Sb}_{2} \mathrm{Te}_{3} \mathrm{SLs}$, which consist of an insulator and a Tl exhibit a topologically protected spin diffusion length of more than $100 \mu \mathrm{m}$ at room temperature. Using TbFeCo electrodes connected in series, a 15 -fold increase in the spin current was possible by accumulating spin between the electrodes by using a SL with 12 periods. The results suggest an exponential increase in spin amplification with the number of SL bilayers. Indeed, if the number of SL periods could be stacked to micrometer thicknesses, a large amount of spin could be accumulated not only in the plane of the electrodes but also within the 3D SL, and this will lead to a large spin current. We previously demonstrated that a cross-point device using the same SL can accumulate spins at zero current for a short period after flowing current perpendicular to the surface ${ }^{22}$. This topologically protected long spin diffusion length is very appealing for spintronics device designers only one spin-injection source (battery) is required, and this will substantially simplify spintronics device designs. The short diffusion lengths of existing spin transport media requires each spintronic device, such as MRAM, to have its own spin current source. Thus, a large number of charge current lines need to be integrated into the device and this severely complicates the circuit design. Moreover, this multiple spin source approach wastes energy. In 
contrast, if one uses the topologically protected long spin diffusion length media, which we demonstrated here, a single spin generator or battery can supply spin current to all spintronic components. This will simplify the circuit design and lower the device energy consumption. For this reason, we believe that NI-TI SLs will become indispensable spin transport media and enable the practical and efficient spintronics devices. In addition, the spin current supplied from the spin generator and rectifier, which we demonstrated here, may also open a new field of spintronics in quantum computation.

\section{METHODS}

Materials and devices

Spin transport devices were fabricated on sapphire substrates, using a sequence of metal stencil masks, After forming a $40 \mathrm{~nm}$-thick amorphous $\mathrm{Si}$ film at room temperature, a $5 \mathrm{~nm}$-thick $\mathrm{Sb}_{2} \mathrm{Te}_{3}$ seed layer, $\mathrm{Ge}_{2} \mathrm{Te}_{2}-\mathrm{Sb}_{2} \mathrm{Te}_{3} \mathrm{SLs}$ were deposited by R.F.-magnetron sputtering at $480 \mathrm{~K}$, using a GeTeS $(0.45$ : $0.52: 0.03)$ and a SbTe$(0.3: 0.7)$ target, respectively. Due to the presence of a $\mathrm{Sb}_{2} \mathrm{Te}_{3}$ seed layer, the $\mathrm{Sb}_{2} \mathrm{Te}_{3}(0001)$ axis and $\mathrm{Ge}_{2} \mathrm{Te}_{2}(111)$ axis were aligned perpendicular to the substrate allowing the SLs to be grown by vdW epitaxy at a couple of hundred degrees above room temperature. Doping (3\%-S) is effective to hold the Ferro-phase stably even at room temperature ${ }^{28,38}$. The standard thicknesses of each material were $0.8 \mathrm{~nm}\left(\mathrm{Ge}_{2} \mathrm{Te}_{2}\right)$ and $1.0 \mathrm{~nm}$ $\left(\mathrm{Sb}_{2} \mathrm{Te}_{3}\right)$, respectively. To protect the films from oxidation during the spin transport measurements, additional $4 \mathrm{QLs}$ were deposited on top of the $\mathrm{SL}$ (total 5QLs). Finally, using other metal masks, TbFeCo $(22.79: 67.17: 10.04)$ electrodes for spin injection and Pt electrodes for detection were separately fabricated on the film surface by sputtering. The electrode widths were $80 \mu \mathrm{m}$ and the distances between the injector and the Pt electrodes were designed at $L=100,200,300$, and $400 \mu \mathrm{m}$ for Device-A. On the other hand, the $L$ was fixed to $200 \mu \mathrm{m}$ for Device-B, C, and D. The electrode thicknesses of Pt and TbFeCo were 70 and $50 \mathrm{~nm}$, respectively. The AMR of the TbFeCo film with a $50 \mathrm{~nm}$ thickness was shown in Supplementary Fig. 1. The resistances between the contact pads in the TbFeCo electrode and in the Pt electrode were about $1.2-4.0$ and $0.2 \sim 0.3 \mathrm{k} \Omega$, respectively. On the other hand, the resistances of the SLs were $1.3 \sim 6.1 \mathrm{k} \Omega$ at around the electrodes.

Simulations. The ab initio density functional theory (DFT) code CASTEP was used to calculate electronic band structures. The generalized gradient approximation of Perdew-Burke-Ernzerhof was used ${ }^{39}$. A $7 \times 7 \times 1$ Monkhorst-Pack grid was utilized for Brillouin zone integrations along using ultrasoft pseudopotentials; a cutoff energy of $230 \mathrm{eV}$ was used ${ }^{40}$. The vdW forces was included using the DFT-D correction proposed by Tkatchenko and Scheffler ${ }^{41}$. The WIEN2k code was also used for spin orbit coupling effect ${ }^{42}$. A value of $R_{\mathrm{MT}} K_{\max }=7.0$ was used for the plane-wave component between augmentation spheres. Monkhorst-Pack grids $(7 \times 7 \times 1)$ were used for Brillouin zone integrations based upon a convergence study carried out using a GeTe/Sb $\mathrm{Te}_{3}$ heterostructure. The energy convergence criterion used was $0.1 \mathrm{mRy}$.

Device measurement. ISHE measurements were performed at room temperature $(300 \mathrm{~K})$ in vacuum using a Hall resistance measurement system (8400 series) from TOYO Corp. The spin signals were measured ten times and then averaged for every $0.01 \mathrm{~T}$ magnetic field step between -0.55 and $+0.55 \mathrm{~T}$.

\section{DATA AVAILABILITY}

The raw data used in this study are available upon reasonable request to the corresponding author.

Received: 11 March 2020; Accepted: 12 June 2020;

Published online: 10 July 2020

\section{REFERENCES}

1. Kang, S. H. Emerging materials and devices in spintronic integrated circuits for energy-smart mobile computing and connectivity. Acta Mater. 61, 952-973 (2013).

2. Apalkov, D., Dieny, B. \& Slaughter, J. M. Magnetoresistive random access memory. Proc. IEEE 104, 1796-1830 (2016).
3. Amiri, P. K. et al. Electric-field-controlled magnetoelectric RAM: progress, challenges, and scaling. IEEE Trans. Magn. 51, 3401507 (2015).

4. Shiota, Y. et al. Evaluation of write error rate for voltage-driven dynamic magnetization switching in magnetic tunnel junctions with perpendicular magnetization. Appl. Phys. Express 9, 013001 (2016).

5. Monsma, D. J., Lodder, J. C., Popma, Th. J. A. \& Dieny, B. Perpendicular hot electron spin-valve effect in a new magnetic field sensor: the spin-valve transistor. Phys. Rev. Lett. 74, 5260-5263 (1995).

6. Sugawara, S. \& Tanaka, M. A spin metal-oxide-semiconductor field-effect transistor using half-metallic-ferromagnet contacts for the source and drain. Appl. Phys. Lett. 84, 2307-2309 (2004).

7. Parkin, S. S. P., Hayashi, M. \& Thomas, L. Magnetic domain-wall racetrack memory. Science 320, 190-194 (2008).

8. Bhatti, S. et al. Spintronics based random access memory: a review. Mater. Today 20, 530-548 (2017).

9. Goto, H. et al. Gate control of spin transport in multilayer graphene. Appl. Phys. Lett. 92, 212110 (2008).

10. Bass, J. \& Pratt, W. P. Jr. Spin-diffusion lengths in metals and alloys, and spinflipping at metal/metal Interfaces: an experimentalist's critical review. J. Phys. Condens. Matter 19, 183201-183602 (2007).

11. Moore, J. E. The birth of topological insulators. Nature 464, 194-198 (2010).

12. Hasan, M. Z. \& Kane, C. L. Colloquium: topological Insulators. Rev. Mod. Phys. 82, 3045-3067 (2010).

13. Qi, X.-L. \& Zhang, S.-C. Topological insulators and superconductors. Rev. Mod. Phys. 83, 1075-1109 (2011).

14. Zhang, $\mathrm{H}$. et al. Topological insulators in $\mathrm{Bi}_{2} \mathrm{Se}_{3}, \mathrm{Bi}_{2} \mathrm{Te}_{3}$ and $\mathrm{Sb}_{2} \mathrm{Te}_{3}$ with a single Dirac cone on the surface. Nat. Phys. 5, 438-442 (2009).

15. Bansil, A., Lin, H. \& Das, T. Colloquium: topological band theory. Rev. Mod. Phys. 88, 021004 (2016).

16. Armitage, N. P., Mele, E. J. \& Vishwanath, A. Weyl and Dirac semimetals in threedimensional solids. Rev. Mod. Phys. 90, 015001 (2018).

17. Simpson, R. et al. Interfacial phase-change memory. Nat. Nanotechnol. 6, 501-505 (2011).

18. Tominaga, J., Kolobov, A. V., Fons, P., Murakami, S. \& Nakano, T. Ferroelectric order control of the Dirac-semimetal phase in $\mathrm{GeTe}-\mathrm{Sb}_{2} \mathrm{Te}_{3}$ superlattices. Adv. Mater. Interfaces 1, 1300027 (2014).

19. Tominaga, J. et al. Giant multiferroic effects in topological GeTe- $\mathrm{Sb}_{2} \mathrm{Te}_{3}$ superlattices. Sci. Technol. Adv. Mater. 15, 014402 (2015).

20. Takagaki, Y., Saito, Y. \& Tominaga, J. Manipulation of the presence of helical surface states of topological insulators using $\mathrm{Sb}_{2} \mathrm{Te}_{3}-\mathrm{GeTe}$ superlattices. Appl. Phys. Lett. 108, 112102 (2016).

21. Nguyen, T.-A. et al. Topological states and phase transitions in $\mathrm{Sb}_{2} \mathrm{Te}_{3}-\mathrm{GeTe}$ multilayers. Sci. Rep. 6, 27716 (2016).

22. Tominaga, J. et al. A magnetoresistance induced by a nonzero berry phase in GeTe/ $\mathrm{Sb}_{2} \mathrm{Te}_{3}$ chalcogenide superlattices. Adv. Funct. Mater. 27, 1702243 (2017).

23. Tominaga, J. et al. Role of $\mathrm{Ge}$ switch in phase transition: approach using atomically controlled GeTe/ $\mathrm{Sb}_{2} \mathrm{Te}_{3}$ superlattice. Jpn J. Appl. Phys. 47, 5763-5766 (2008).

24. Tominaga, J., Simpson, R. E., Fons, P. \& Kolobov, A. V. Electrical-field induced giant magnetoresistivity in (non-magnetic) phase change films. Appl. Phys. Lett. 99, 152105 (2011).

25. Kim, J. et al. Weyl node assisted conductivity switch in interfacial phase-change memory with van der Waals interfaces. Phys. Rev. B 96, 235304 (2017).

26. Eremeev, S. V., Rusinov, I. P., Echenique, P. M. \& Chulkov, E. V. Temperature-driven topological quantum phase transitions in a phase-change material $\mathrm{Ge}_{2} \mathrm{Sb}_{2} \mathrm{Te}_{5}$. Sci. Rep. 6, 38799 (2016).

27. Tominaga, J. Topological memory using phase-change materials. MRS Bull. 43, 347-351 (2018).

28. Tominaga, J., Sumi, S. \& Awano, H. Intermixing suppression through the interface in $\mathrm{GeTe} / \mathrm{Sb}_{2} \mathrm{Te}_{3}$ superlattice. Appl. Phys. Express 13, 075503 (2020).

29. Yu, X. \& Robertson, J. Modeling of switching mechanism in GeSbTe chalcogenide superlattices. Sci. Rep. 5, 12612 (2015).

30. Sa, B., Zhou, J., Sun, Z., Tominaga, J. \& Ahuja, R. Topological Insulating GeTe/ $\mathrm{Sb}_{2} \mathrm{Te}_{3}$ Phase-Change Superlattice. Phys. Rev. Lett. 109, 096802 (2012).

31. Naoe, M., Kitamura, N. \& Hirata, T. Preparation of amorphous Tb-Fb-Co thin films by plasma-free high-rate sputtering. J. Appl. Phys. 61, 3337 (1987).

32. Lee, J.-W., Shieh, H.-P. D., Kryder, M. H. \& Laughlin, D. E. The effect of deposition conditions on microstructure and magnetic properties of TbFeCo. J. Appl. Phys. 63, 3626 (1988).

33. Hirsh, J. E. Spin Hall Effect. Phys. Rev. Lett. 83, 1834-1837 (1999).

34. Saitoh, E., Ueda, M., Miyajima, H. \& Tatara, G. Conversion of spin current into charge current at room temperature: Inverse spin-Hall effect. Appl. Phys. Lett. 88, 182509 (2006).

35. Maekawa S., Valenzuela S. O., Saitoh E. \& Kimura T. (eds). Spin Current (Oxford Science, 2012).

36. Datta, S. Proposal for a "spin capacitor". Appl. Phys. Lett. 87, 013115-1-3-3 (2005). 
37. Semenov, Y. G., Zavada, J. M. \& Kim, K. W. Graphene spin capacitor for magnetic field scanning. Appl. Phys. Lett. 97, 013106-1-3-3 (2010).

38. Tominaga J., Miyata N., Kamata Y. \& Kunishima I. Japanese Patent Application No. 2018-130365, (2018).

39. Perdew, J. P., Burke, K. \& Ernzerhof, M. Generalized gradient approximation made simple. Phys. Rev. Lett. 77, 3865-3868 (1996)

40. Monkhorst, H. J. \& Pack, J. D. Special points for Brillouin-zone integrations. Phys. Rev. B 13, 5188-5192 (1976).

41. Tkatchenko, A. \& Scheffler, M. Accurate molecular van der Waals interactions from ground-state electron density and free- atom reference data. Phys. Rev. Lett. 102, 073005-1-4 (2009).

42. Schwarz, K. \& Blaha, P. Solid state calculations using WIEN2k. Comput. Mater. Sci. 28, 259-273 (2003)

\section{ACKNOWLEDGEMENTS}

The work was fully supported by grant number JPMJCR14F1 of the Japan Science and Technology Agency(JST/CREST). We thank K. Koyama of Toray Research Center for TEM measurement.

\section{AUTHOR CONTRIBUTIONS}

H.A., N.M., S.M., and J.T. coordinated the project. J.T. fabricated the superlattice devices. S S., H.A., and J.T. measured the spin signals. S.M. contributed the theoretical parts. J.T. wrote the manuscript.

\section{COMPETING INTERESTS}

The authors declare no competing interests.

\section{ADDITIONAL INFORMATION}

Supplementary information is available for this paper at https://doi.org/10.1038/ s41699-020-0157-8.

Correspondence and requests for materials should be addressed to J.T.

Reprints and permission information is available at http://www.nature.com/ reprints

Publisher's note Springer Nature remains neutral with regard to jurisdictional claims in published maps and institutional affiliations.
Open Access This article is licensed under a Creative Commons Attribution 4.0 International License, which permits use, sharing, adaptation, distribution and reproduction in any medium or format, as long as you give appropriate credit to the original author(s) and the source, provide a link to the Creative Commons license, and indicate if changes were made. The images or other third party material in this article are included in the article's Creative Commons license, unless indicated otherwise in a credit line to the material. If material is not included in the article's Creative Commons license and your intended use is not permitted by statutory regulation or exceeds the permitted use, you will need to obtain permission directly from the copyright holder. To view a copy of this license, visit http://creativecommons. org/licenses/by/4.0/.

(c) The Author(s) 2020 